\title{
Fourier series of sums of products of Genocchi functions and their applications
}

\author{
Taekyun Kimª,b, Dae San Kimc, Gwan-Woo Jang ${ }^{b}$, Jongkyum Kwon ${ }^{\text {d,* }}$ \\ ${ }^{a}$ Department of Mathematics, College of Science, Tianjin Polytechnic University, Tianjin 300160, China. \\ ${ }^{b}$ Department of Mathematics, Kwangwoon University, Seoul, 139-701, Republic of Korea. \\ ${ }^{c}$ Department of Mathematics, Sogang University, Seoul, 121-742, Republic of Korea. \\ ${ }^{d}$ Department of Mathematics Education and RINS, Gyeongsang National University, Jinju, Gyeongsangnamdo, 52828, Republic of \\ Korea.
}

\begin{abstract}
Recently, Luo introduced Fourier expansions of Apostol-Bernoulli, Apostol-Euler and Genocchi polynomials and investigated some interesting identities and properties of these polynomials by using Fourier series. In this paper, we consider three types of functions given by sums of products of Genocchi functions and derive their Fourier series expansions. In addition, we will express each of them in terms of Bernoulli functions. (C)2017 All rights reserved.
\end{abstract}

Keywords: Fourier series, Genocchi functions, Genocchi polynomials. 2010 MSC: 11B83, 42A16.

\section{Introduction}

Let $G_{m}(x)$ be the Genocchi polynomials given by the generating function (see $[2,6,7,9-11,21]$ )

$$
\frac{2 t}{e^{t}+1} e^{x t}=\sum_{m=0}^{\infty} G_{m}(x) \frac{t^{m}}{m !} .
$$

For $x=0, G_{m}=G_{m}(0)$ are called Genocchi numbers. The first few Genocchi polynomials are as follows:

$$
\begin{aligned}
& \mathrm{G}_{0}(x)=0, \mathrm{G}_{1}(x)=1, \mathrm{G}_{2}(x)=2 x-1, \\
& \mathrm{G}_{3}(x)=3 x^{2}-3 x, \mathrm{G}_{4}(x)=4 x^{3}-6 x^{2}+1, \\
& \mathrm{G}_{5}(x)=5 x^{4}-10 x^{3}+5 x, \mathrm{G}_{6}(x)=6 x^{5}-15 x^{4}+15 x^{2}-3 \\
& \mathrm{G}_{7}(x)=7 x^{6}-21 x^{5}+35 x^{3}-21 x .
\end{aligned}
$$

\footnotetext{
*Corresponding author

Email addresses: tkkim@kw.ac.kr (Taekyun Kim), dskim@sogang.ac.kr (Dae San Kim), jgw5687@naver.com (Gwan-Woo Jang), mathkjk26@gnu.ac.kr (Jongkyum Kwon)
}

doi:10.22436/jnsa.010.04.31 
From the relation $G_{m}(x)=m E_{m-1}(x)$, we see that

$$
\operatorname{deg} G_{m}(x)=m-1, G_{m}=m E_{m-1}, \quad G_{0}=0, G_{1}=1, G_{2 m+1}=0, G_{2 m+1} \neq 0,
$$

where $m \geqslant 1$. Also,

$$
\frac{d}{d x} G_{m}(x)=m G_{m-1}(x),(m \geqslant 1), \quad G_{m}(x+1)+G_{m}(x)=2 m x^{m-1},(m \geqslant 0) .
$$

From (1.1), we have

$$
\begin{aligned}
G_{m}(1)+G_{m}(0) & =2 \delta_{m, 1},(m \geqslant 0), \\
\int_{0}^{1} G_{m}(x) d x & =\frac{1}{m+1}\left(G_{m+1}(1)-G_{m+1}(0)\right) \\
& =\frac{2}{m+1}\left(-G_{m+1}(0)+\delta_{m, 0}\right) \\
& = \begin{cases}0, & \text { for m even, } \\
-\frac{2}{m+1} G_{m+1}, & \text { for } m \text { odd }\end{cases}
\end{aligned}
$$

For any real number $x$, we let

$$
<x>=x-[x] \in[0,1)
$$

denote the fractional part of $x$.

Here we will consider the following three types of sums of products of Genocchi functions and derive their Fourier series expansions. Further, we will express each of them in terms of Bernoulli functions $\mathrm{B}_{\mathrm{m}}(<x>)$.

(1) $\alpha_{m}(<x>)=\sum_{k=0}^{m} G_{k}(<x>) G_{m-k}(<x>),(m \geqslant 3)$;

(2) $\beta_{m}(<x>)=\sum_{k=0}^{m} \frac{1}{k !(m-k) !} G_{k}(<x>) G_{m-k}(<x>),(m \geqslant 3)$;

(3) $\gamma_{m}(<x>)=\sum_{k=1}^{m-1} \frac{1}{k(m-k)} G_{k}(<x>) G_{m-k}(<x>),(m \geqslant 3)$.

For elementary facts about Fourier analysis, the reader may refer to any book (for example, see $[8,17,18$, 22]).

As to $\gamma_{m}(<x>)$, we note that the polynomial identity (1.2) follows immediately from Theorems 4.1 and 4.2, which is in turn derived from the Fourier series expansion of $\gamma_{m}(<x>)$,

$$
\sum_{k=1}^{m-1} \frac{1}{k(m-k)} G_{k}(x) G_{m-k}(x)=-\frac{4}{m} \sum_{k=0}^{m-2}\left(\begin{array}{c}
m \\
k
\end{array}\right) \frac{G_{m-k}}{m-k} B_{k}(x), \quad(m \geqslant 3) .
$$

The obvious polynomial identities can be derived also for $\alpha_{m}(\langle x\rangle)$ and $\left.\beta_{m}(<x\rangle\right)$ from Theorems 2.1 and 2.2, and Theorems 3.1 and 3.2, respectively. It is noteworthy that from the Fourier series expansion of the function $\sum_{k=1}^{m-1} \frac{1}{k(m-k)} B_{k}(<x>) B_{m-k}(<x>)$ we can derive the following corresponding polynomial identity:

$$
\begin{aligned}
& \sum_{k=1}^{m-1} \frac{1}{k(m-k)} B_{k}(x) B_{m-k}(x) \\
& \quad=\frac{2}{m^{2}}\left(B_{m}+\frac{1}{2}\right)+\frac{2}{m} \sum_{k=1}^{m-2} \frac{1}{m-k}\left(\begin{array}{c}
m \\
k
\end{array}\right) B_{m-k} B_{k}(x)+\frac{2}{m} H_{m-1} B_{m}(x), \quad(m \geqslant 2) .
\end{aligned}
$$

Simple modification of (1.3) yields a slightly different version of the well-known Miki's identity (see $[1,5,19,20])$ :

$$
\sum_{k=1}^{m-1} \frac{1}{2 k(2 m-2 k)} B_{2 k} B_{2 m-2 k}=\frac{1}{m} \sum_{k=1}^{m} \frac{1}{2 k}\left(\begin{array}{c}
2 m \\
2 k
\end{array}\right) B_{2 k} B_{2 m-2 k}+\frac{1}{m} H_{2 m-1} B_{2 m}, \quad(m \geqslant 2) .
$$


With $\bar{B}_{m}=\left(\frac{1-2^{m-1}}{2^{m-1}}\right) B_{m}=\left(2^{1-m}-1\right) B_{m}=B_{m}\left(\frac{1}{2}\right)$, from (1.3) we can also derive

$$
\sum_{k=1}^{m-1} \frac{1}{2 k(2 m-2 k)} \bar{B}_{2 k} \bar{B}_{2 m-2 k}=\frac{1}{m} \sum_{k=1}^{m} \frac{1}{2 k}\left(\begin{array}{c}
2 m \\
2 k
\end{array}\right) B_{2 k} \bar{B}_{2 m-2 k}+\frac{1}{m} H_{2 m-1} \bar{B}_{2 m}, \quad(m \geqslant 2),
$$

which is the Faber-Pandharipande-Zagier identity (see [4]). Some related works can be found in [3, 12-16].

\section{The function $\alpha_{m}(<x>)$}

Let $\alpha_{m}(x)=\sum_{k=1}^{m-1} G_{k}(x) G_{m-k}(x),(m \geqslant 3)$. Note here that $\operatorname{deg} \alpha_{m}(x)=m-2$. Then we will consider the function

$$
\alpha_{m}(<x>)=\sum_{k=1}^{m-1} G_{k}(<x>) G_{m-k}(<x>),(m \geqslant 3),
$$

defined on $(-\infty, \infty)$, which is periodic with period 1. The Fourier series of $\alpha_{m}(<x>)$ is

$$
\sum_{n=-\infty}^{\infty} A_{n}^{(m)} e^{2 \pi i n x}
$$

where

$$
A_{n}^{(m)}=\int_{0}^{1} \alpha_{m}(<x>) e^{-2 \pi i n x} d x=\int_{0}^{1} \alpha_{m}(x) e^{-2 \pi i n x} d x
$$

Before proceeding further, we note the following.

$$
\begin{aligned}
\alpha_{m}^{\prime}(x) & =\sum_{k=1}^{m-1}\left(k G_{k-1}(x) G_{m-k}(x)+(m-k) G_{k}(x) G_{m-k-1}(x)\right) \\
& =\sum_{k=2}^{m-1} k G_{k-1}(x) G_{m-k}(x)+\sum_{k=1}^{m-2}(m-k) G_{k}(x) G_{m-k-1}(x) \\
& =\sum_{k=1}^{m-2}(k+1) G_{k}(x) G_{m-1-k}(x)+\sum_{k=1}^{m-2}(m-k) G_{k}(x) G_{m-1-k}(x) \\
& =(m+1) \sum_{k=1}^{m-2} G_{k}(x) G_{m-1-k}(x)=(m+1) \alpha_{m-1}(x) .
\end{aligned}
$$

From this, we have $\left(\frac{\alpha_{m+1}(x)}{m+2}\right)^{\prime}=\alpha_{m}(x)$,

$$
\begin{aligned}
\int_{0}^{1} \alpha_{m}(x) d x & =\frac{1}{m+2}\left(\alpha_{m+1}(1)-\alpha_{m+1}(0)\right) \\
\alpha_{m}(1)-\alpha_{m}(0) & =\sum_{k=1}^{m-1}\left(G_{k}(1) G_{m-k}(1)-G_{k}(0) G_{m-k}(0)\right) \\
& =\sum_{k=1}^{m-1}\left(\left(-G_{k}(0)+2 \delta_{k, 1}\right)\left(-G_{m-k}(0)+2 \delta_{m-k, 1}\right)-G_{k}(0) G_{m-k}(0)\right) \\
& =\sum_{k=1}^{m-1}\left(-2 G_{k}(0) \delta_{m-k, 1}-2 \delta_{k, 1} G_{m-k}(0)+4 \delta_{k, 1} \delta_{m-k, 1}\right) \\
& =-4 G_{m-1}(0)+4 \delta_{m-1,1}=-4 G_{m-1}(0) .
\end{aligned}
$$


Thus

$$
\alpha_{m}(1)=\alpha_{m}(0) \Longleftrightarrow G_{m-1}(0)=0,(m \geqslant 3) \Longleftrightarrow m \text { is even, }(m \geqslant 3) .
$$

Also,

$$
\int_{0}^{1} \alpha_{m}(x) d x=\frac{-4}{m+2} G_{m}
$$

Now, we would like to determine the Fourier coefficients $A_{n}^{(m)}$.

Case $1: n \neq 0$.

$$
\begin{aligned}
A_{n}^{(m)} & =\int_{0}^{1} \alpha_{m}(x) e^{-2 \pi i n x} d x \\
& =-\frac{1}{2 \pi i n}\left[\alpha_{m}(x) e^{-2 \pi i n x}\right]_{0}^{1}+\frac{1}{2 \pi i n} \int_{0}^{1} \alpha_{m}^{\prime}(x) e^{-2 \pi i n x} d x \\
& =-\frac{1}{2 \pi i n}\left(\alpha_{m}(1)-\alpha_{m}(0)\right)+\frac{m+1}{2 \pi i n} \int_{0}^{1} \alpha_{m-1}(x) e^{-2 \pi i n x} d x \\
& =\frac{m+1}{2 \pi i n} A_{n}^{(m-1)}+\frac{2}{\pi i n} G_{m-1} \\
& =\frac{m+1}{2 \pi i n}\left(\frac{m}{2 \pi i n} A_{n}^{(m-2)}+\frac{2}{\pi i n} G_{m-2}\right)+\frac{2}{\pi i n} G_{m-1} \\
& =\frac{(m+1) m}{(2 \pi i n)^{2}} A_{n}^{(m-2)}+\frac{m+1}{2 \pi i n} \frac{2}{\pi i n} G_{m-2}+\frac{2}{\pi i n} G_{m-1} \\
& =\frac{(m+1) m}{(2 \pi i n)^{2}}\left(\frac{m-1}{2 \pi i n} A_{n}^{(m-3)}+\frac{2}{\pi i n} G_{m-3}\right)+\frac{m+1}{2 \pi i n} \frac{2}{\pi i n} G_{m-2}+\frac{2}{\pi i n} G_{m-1} \\
& =\frac{(m+1) m(m-1)}{(2 \pi i n)^{3}} A_{n}^{(m-3)}+\frac{(m+1) m}{(2 \pi i n)^{2}} \frac{2}{\pi i n} G_{m-3}+\frac{m+1}{2 \pi i n} \frac{2}{\pi i n} G_{m-2}+\frac{2}{\pi i n} G_{m-1} \\
& \vdots \\
& =\frac{(m+1)_{m-3}}{(2 \pi i n)^{m-3}} A_{n}^{(3)}+\sum_{k=1}^{m-3} \frac{(m+1)_{k-1}}{(2 \pi i n)^{k-1}} \frac{2}{\pi i n} G_{m-k} \\
= & -4 \frac{(m+1)_{m-3}}{(2 \pi i n)^{m-2}}+4 \sum_{k=1}^{m-3} \frac{(m+1)_{k-1}}{(2 \pi i n)^{k}} G_{m-k} \\
= & 4 \sum_{k=1}^{m-2} \frac{(m+1)_{k-1}}{(2 \pi i n)^{k}} G_{m-k} \\
= & \frac{4}{m+2} \sum_{k=1}^{m-2} \frac{(m+2)_{k}}{(2 \pi i n)^{k}} G_{m-k} \\
&
\end{aligned}
$$

where $A_{n}^{(3)}=\int_{0}^{1} \alpha_{3}(x) e^{-2 \pi i n x} d x=\int_{0}^{1}(4 x-2) e^{-2 \pi i n x} d x=-\frac{2}{\pi i n}$.

Case 2: $\mathrm{n}=0$. We have

$$
A_{0}^{(m)}=\int_{0}^{1} \alpha_{m}(x) d x=\frac{-4}{m+2} G_{m}
$$

and $\alpha_{m}(<x>),(m \geqslant 3)$ is piecewise $C^{\infty}$. In addition, $\alpha_{m}(<x>)$ is continuous for all even positive integers $m \geqslant 4$ and discontinuous with jump discontinuities at integers for all odd positive integers $m \geqslant 3$.

We now recall the following facts about Bernoulli functions $B_{n}(<x>)$ : 
(1) for $m \geqslant 2$,

$$
B_{m}(<x>)=-m ! \sum_{n=-\infty, n \neq 0}^{\infty} \frac{e^{2 \pi i n x}}{(2 \pi i n)^{m}}
$$

(2) for $m=1$,

$$
-\sum_{n=-\infty, n \neq 0}^{\infty} \frac{e^{2 \pi i n x}}{2 \pi i n}= \begin{cases}B_{1}(<x>), & \text { for } x \notin \mathbb{Z}, \\ 0, & \text { for } x \in \mathbb{Z} .\end{cases}
$$

Assume first that $m$ is an even positive integer $\geqslant 4$. Then $\alpha_{m}(1)=\alpha_{m}(0)$.

$\alpha_{m}(<x>)$ is piecewise $C^{\infty}$, and continuous. So the Fourier series of $\alpha_{m}(<x>)$ converges uniformly to $\alpha_{m}(<x>)$, and

$$
\begin{aligned}
& \alpha_{m}(<x>)=\frac{-4}{m+2} G_{m}+\sum_{n=-\infty, n \neq 0}^{\infty}\left(\frac{4}{m+2} \sum_{k=1}^{m-2} \frac{(m+2)_{k}}{(2 \pi i n)^{k}} G_{m-k}\right) e^{2 \pi i n x} \\
& =\frac{-4}{m+2} G_{m}-\frac{4}{m+2} \sum_{k=1}^{m-2}\left(\begin{array}{c}
m+2 \\
k
\end{array}\right) G_{m-k}\left(\begin{array}{c}
-k ! \sum_{n=-\infty, n \neq 0}^{\infty} \frac{e^{2 \pi i n x}}{(2 \pi i n)^{k}}
\end{array}\right) \\
& =\frac{-4}{m+2} G_{m}-\frac{4}{m+2} \sum_{k=2}^{m-2}\left(\begin{array}{c}
m+2 \\
k
\end{array}\right) G_{m-k} B_{k}(<x>) \\
& -\frac{4}{m+2}\left(\begin{array}{c}
m+2 \\
1
\end{array}\right) G_{m-1} \cdot \begin{cases}B_{1}(<x>), & \text { for } x \notin \mathbb{Z}, \\
0, & \text { for } x \in \mathbb{Z}\end{cases} \\
& =\frac{-4}{m+2} G_{m}-\frac{4}{m+2} \sum_{k=2}^{m-2}\left(\begin{array}{c}
m+2 \\
k
\end{array}\right) G_{m-k} B_{k}(<x>) \\
& =\frac{-4}{m+2} \sum_{k=0, k \neq 1}^{m-2}\left(\begin{array}{c}
m+2 \\
k
\end{array}\right) G_{m-k} B_{k}(<x>)
\end{aligned}
$$

for all $x \in(-\infty, \infty)$. Hence we obtain the following theorem.

Theorem 2.1. Let $\mathrm{m}$ be an even integer with $\mathrm{m} \geqslant 4$. Then we have the following:

(a) $\sum_{k=1}^{m-1} G_{k}(<x>) G_{m-k}(<x>)$ has the Fourier series expansion

$$
\sum_{k=1}^{m-1} G_{k}(<x>) G_{m-k}(<x>)=-\frac{4}{m+2} G_{m}+\sum_{n=-\infty, n \neq 0}^{\infty}\left(\frac{4}{m+2} \sum_{k=1}^{m-2} \frac{(m+2)_{k}}{(2 \pi i n)^{k}} G_{m-k}\right) e^{2 \pi i n x}
$$

for all $x \in(-\infty, \infty)$, where the convergence is uniform;

(b)

$$
\sum_{k=1}^{m-1} G_{k}(<x>) G_{m-k}(<x>)=-\frac{4}{m+2} \sum_{k=0, k \neq 1}^{m-2}\left(\begin{array}{c}
m+2 \\
k
\end{array}\right) G_{m-k} B_{k}(<x>)
$$

for all $\mathrm{x} \in(-\infty, \infty)$, where $\left.\mathrm{B}_{\mathrm{k}}(<\mathrm{x}\rangle\right)$ is the Bernoulli function.

Assume next that $m$ is an odd integer $\geqslant 3$. Then $\alpha_{m}(1) \neq \alpha_{m}(0)$, and hence $\alpha_{m}(<x>)$ is piecewise $\mathrm{C}^{\infty}$, and discontinuous with jump discontinuities at integers. The Fourier series of $\alpha_{\mathrm{m}}(<x>)$ converges pointwise to $\alpha_{m}(<x>$ ), for $x \notin \mathbb{Z}$, and converges to

$$
\frac{1}{2}\left(\alpha_{m}(0)+\alpha_{m}(1)\right)=\alpha_{m}(0)-2 G_{m-1}
$$

for $x \in \mathbb{Z}$. Thus, we get the following theorem. 
Theorem 2.2. Let $\mathrm{m}$ be an odd positive integer with $\mathrm{m} \geqslant 3$. Then we have the following:

(a)

$$
\sum_{n=-\infty, n \neq 0}^{\infty}\left(\frac{4}{m+2} \sum_{k=1}^{m-2} \frac{(m+2)_{k}}{(2 \pi i n)^{k}} G_{m-k}\right) e^{2 \pi i n x}= \begin{cases}\sum_{k=1}^{m-1} G_{k}(<x>) G_{m-k}(<x>), & \text { for } x \notin \mathbb{Z}, \\ \sum_{k=1}^{m-1} G_{k} G_{m-k}-2 G_{m-1}, & \text { for } x \in \mathbb{Z} ;\end{cases}
$$

(b)

$$
\begin{aligned}
& -\frac{4}{m+2} \sum_{k=1}^{m-2}\left(\begin{array}{c}
m+2 \\
k
\end{array}\right) G_{m-k} B_{k}(<x>)=\sum_{k=1}^{m-1} G_{k}(<x>) G_{m-k}(<x>), \text { for } x \notin \mathbb{Z}, \\
& -\frac{4}{m+2} \sum_{k=2}^{m-2}\left(\begin{array}{c}
m+2 \\
k
\end{array}\right) G_{m-k} B_{k}(<x>)=\sum_{k=1}^{m-1} G_{k} G_{m-k}-2 G_{m-1}, \text { for } x \in \mathbb{Z} .
\end{aligned}
$$

3. The function $\beta_{m}(<x>)$

Let $\beta_{m}(x)=\sum_{k=1}^{m-1} \frac{1}{k !(m-k) !} G_{k}(x) G_{m-k}(x), \quad(m \geqslant 3)$. Then we will consider the function

$$
\beta_{m}(<x>)=\sum_{k=1}^{m-1} \frac{1}{k !(m-k) !} G_{k}(<x>) G_{m-k}(<x>),
$$

defined on $(-\infty, \infty)$, which is periodic with period 1. The Fourier series of $\beta_{m}(<x>)$ is

$$
\sum_{n=-\infty}^{\infty} B_{n}^{(m)} e^{2 \pi i n x}
$$

where

$$
B_{n}^{(m)}=\int_{0}^{1} \beta_{m}(<x>) e^{-2 \pi i n x} d x=\int_{0}^{1} \beta_{m}(x) e^{-2 \pi i n x} d x .
$$

To proceed further, we observe the following:

$$
\begin{aligned}
\beta_{m}^{\prime}(x) & =\sum_{k=1}^{m-1}\left\{\frac{k}{k !(m-k) !} G_{k-1}(x) G_{m-k}(x)+\frac{m-k}{k !(m-k) !} G_{k}(x) G_{m-k-1}(x)\right\} \\
& =\sum_{k=2}^{m-1} \frac{1}{(k-1) !(m-k) !} G_{k-1}(x) G_{m-k}(x)+\sum_{k=1}^{m-2} \frac{1}{k !(m-k-1) !} G_{k}(x) G_{m-k-1}(x) \\
& =2 \sum_{k=1}^{m-2} \frac{1}{k !(m-1-k) !} G_{k}(x) G_{m-1-k}(x) \\
& =2 \beta_{m-1}(x) .
\end{aligned}
$$

From this, we get $\left(\frac{\beta_{m+1}(x)}{2}\right)^{\prime}=\beta_{m}(x)$, and

$$
\begin{aligned}
\int_{0}^{1} \beta_{m}(x) d x & =\frac{1}{2}\left(\beta_{m+1}(1)-\beta_{m+1}(0)\right) \\
\beta_{m}(1)-\beta_{m}(0) & =\sum_{k=1}^{m-1} \frac{1}{k !(m-k) !}\left(G_{k}(1) G_{m-k}(1)-G_{k}(0) G_{m-k}(0)\right)
\end{aligned}
$$




$$
\begin{aligned}
& =\sum_{k=1}^{m-1} \frac{1}{k !(m-k) !}\left\{\left(-G_{k}(0)+2 \delta_{k, 1}\right) \times\left(-G_{m-k}(0)+2 \delta_{m-k, 1}\right)-G_{k}(0) G_{m-k}(0)\right\} \\
& =\sum_{k=1}^{m-1} \frac{1}{k !(m-k) !}\left(-2 G_{k}(0) \delta_{m-k, 1}-2 \delta_{k, 1} G_{m-k}(0)+4 \delta_{k, 1} \delta_{m-k, 1}\right) \\
& =\frac{1}{(m-1) !}\left(-2 G_{m-1}(0)-2 G_{m-1}(0)+4 \delta_{m-1,1}\right) \\
& =-\frac{4}{(m-1) !} G_{m-1} .
\end{aligned}
$$

So, for $m \geqslant 3, \beta_{m}(1)-\beta_{m}(0)=-\frac{4}{(m-1) !} G_{m-1}$.

Hence $\beta_{\mathrm{m}}(1)=\beta_{\mathrm{m}}(0) \Leftrightarrow \mathrm{G}_{\mathrm{m}-1}=0 \Leftrightarrow \mathrm{m}$ is an even integer with $\mathrm{m} \geqslant 4$. Also,

$$
\int_{0}^{1} \beta_{m}(x) d x=-\frac{2 G_{m}}{m !} .
$$

We are now ready to determine the Fourier coefficients $B_{n}^{(m)}$.

Case $1: n \neq 0$.

$$
\begin{aligned}
& B_{n}^{(m)}=\int_{0}^{1} \beta_{m}(x) e^{-2 \pi i n x} d x \\
&=-\frac{1}{2 \pi i n}\left[\beta_{m}(x) e^{-2 \pi i n x}\right]_{0}^{1}+\frac{1}{2 \pi i n} \int_{0}^{1} \beta_{m}^{\prime}(x) e^{-2 \pi i n x} d x \\
&=-\frac{1}{2 \pi i n}\left(\beta_{m}(1)-\beta_{m}(0)\right)+\frac{1}{\pi i n} \int_{0}^{1} \beta_{m-1}(x) e^{-2 \pi i n x} d x \\
&=\frac{1}{\pi i n} B_{n}^{(m-1)}+\frac{2}{\pi i n} \frac{G_{m-1}}{(m-1) !} \\
&=\frac{1}{\pi i n}\left(\frac{1}{\pi i n} B_{n}^{(m-2)}+\frac{2}{\pi i n} \frac{G_{m-2}}{(m-2) !}\right)+\frac{2}{\pi i n} \frac{G_{m-1}}{(m-1) !} \\
&=\frac{1}{(\pi i n)^{2}} B_{n}^{(m-2)}+\frac{2}{(\pi i n)^{2}} \frac{G_{m-2}}{(m-2) !}+\frac{2}{\pi i n} \frac{G_{m-1}}{(m-1) !} \\
&=\frac{1}{(\pi i n)^{2}}\left(\frac{1}{\pi i n} B_{n}^{(m-3)}+\frac{2}{\pi i n} \frac{G_{m-3}}{(m-3) !}\right)+\frac{2}{(\pi i n)^{2}} \frac{G_{m-2}}{(m-2) !}+\frac{2}{\pi i n} \frac{G_{m-1}}{(m-1) !} \\
&=\frac{1}{(\pi i n)^{3}} B_{n}^{(m-3)}+\frac{2}{(\pi i n)^{3}} \frac{G_{m-3}}{(m-3) !}+\frac{2}{(\pi i n)^{2}} \frac{G_{m-2}}{(m-2) !}+\frac{2}{\pi i n} \frac{G_{m}-1}{(m-1) !} \\
& \vdots \\
&=\frac{1}{(\pi i n)^{m-3}} B_{n}^{(3)}+\sum_{k=1}^{m-3} \frac{2}{(\pi i n)^{k}} \frac{G_{m}-k}{(m-k) !} \\
&=-\frac{1}{(\pi i n)^{m-2}}+\sum_{k=1}^{m-3} \frac{2}{(\pi i n)^{k}} \frac{G_{m-k}}{(m-k) !}=\sum_{k=1}^{m-2} \frac{2}{(\pi i n)^{k}} \frac{G_{m-k}}{(m-k) !}, \\
&
\end{aligned}
$$

where $B_{n}^{(3)}=\int_{0}^{1} \beta_{3}(x) e^{-2 \pi i n x} d x=\int_{0}^{1}(2 x-1) e^{-2 \pi i n x} d x=-\frac{1}{\pi i n}$.

Case 2: $\mathrm{n}=0$.

$$
\mathrm{B}_{0}^{(\mathrm{m})}=\int_{0}^{1} \beta_{\mathrm{m}}(\mathrm{x})=-\frac{2 \mathrm{G}_{\mathrm{m}}}{\mathrm{m} !} .
$$

$\beta_{m}(<x>), \quad(m \geqslant 3)$ is piecewise $C^{\infty}$. In addition, $\beta_{m}(<x>)$ is continuous for all even positive integers $m \geqslant 4$ and discontinuous with jump discontinuities at integers for all odd positive integers $m \geqslant 3$. 
Assume that $m$ is an even positive integer with $m \geqslant 4$. Then $\beta_{m}(1)=\beta_{m}(0) . \beta_{m}(<x>)$ is piecewise $C^{\infty}$, and continuous. Hence the Fourier series of $\beta_{m}(<x>)$ converges uniformly to $\beta_{m}(<x>)$, and

$$
\begin{aligned}
& \beta_{m}(<x>)=-\frac{2 G_{m}}{m !}+\sum_{n=-\infty, n \neq 0}^{\infty}\left(\sum_{k=1}^{m-2} \frac{2}{(\pi i n)^{k}} \frac{G_{m-k}}{(m-k) !}\right) e^{2 \pi i n x} \\
& =-\frac{2 G_{m}}{m !}-\frac{1}{m !} \sum_{k=1}^{m-2} 2^{k+1}\left(\begin{array}{c}
m \\
k
\end{array}\right) G_{m-k} \times\left(-k ! \sum_{n=-\infty, n \neq 0}^{\infty} \frac{e^{2 \pi i n x}}{(2 \pi i n)^{k}}\right) \\
& =-\frac{2 G_{m}}{m !}-\frac{1}{m !} \sum_{k=2}^{m-2} 2^{k+1}\left(\begin{array}{c}
m \\
k
\end{array}\right) G_{m-k} B_{k}(<x>)-\frac{4}{m !}\left(\begin{array}{c}
m \\
1
\end{array}\right) G_{m-1} \times \begin{cases}B_{1}(<x>), & \text { for } x \notin \mathbb{Z}, \\
0, & \text { for } x \in \mathbb{Z}\end{cases} \\
& =-\frac{2 G_{m}}{m !}-\frac{1}{m !} \sum_{k=2}^{m-2} 2^{k+1}\left(\begin{array}{c}
m \\
k
\end{array}\right) G_{m-k} B_{k}(<x>) \\
& =-\frac{1}{m !} \sum_{k=0, k \neq 1}^{m-2} 2^{k+1}\left(\begin{array}{c}
m \\
k
\end{array}\right) G_{m-k} B_{k}(<x>), \text { for all } x \in(-\infty, \infty) .
\end{aligned}
$$

Thus we have the following theorem.

Theorem 3.1. Let $\mathrm{m}$ be an even integer with $\mathrm{m} \geqslant 4$. Then we have the following:

(a) $\sum_{k=1}^{m-1} \frac{1}{k !(m-k) !} G_{k}(<x>) G_{m-k}(<x>)$ has the Fourier series expansion

$$
-\frac{2 G_{m}}{m !}+\sum_{n=-\infty, n \neq 0}^{\infty}\left(\sum_{k=1}^{m-2} \frac{2}{(\pi i n)^{k}} \frac{G_{m-k}}{(m-k) !}\right) e^{2 \pi i n x}
$$

(b)

for all $x \in(-\infty, \infty)$, where the convergence is uniform;

$$
\sum_{k=1}^{m-1} \frac{1}{k !(m-k) !} G_{k}(<x>) G_{m-k}(<x>)=-\frac{1}{m !} \sum_{k=0, k \neq 1}^{m-2} 2^{k+1}\left(\begin{array}{c}
m \\
k
\end{array}\right) G_{m-k} B_{k}(<x>)
$$

for all $\mathrm{x} \in(-\infty, \infty)$, where $\left.\mathrm{B}_{\mathrm{k}}(<\mathrm{x}\rangle\right)$ is the Bernoulli function.

Assume next that $m$ is an odd integer with $m \geqslant 3$. Then, $\beta_{m}(1) \neq \beta_{m}(0)$, and hence $\beta_{m}(<x>)$ is piecewise $C^{\infty}$ and discontinous with jump discontinuities at integers. Thus the Fourier series of $\beta_{\mathrm{m}}(<$ $x>$ ) converges pointwise to $\beta_{m}(<x>)$ for $x \notin \mathbb{Z}$, and converges to

$$
\frac{1}{2}\left(\beta_{m}(0)+\beta_{m}(1)\right)=\beta_{m}(0)-\frac{2}{(m-1) !} G_{m-1}
$$

for $x \in \mathbb{Z}$. Thus we get the following theorem.

Theorem 3.2. Let $\mathrm{m}$ be an odd positive integer with $\mathrm{m} \geqslant 3$. Thus we have the following:

(a)

$$
\sum_{n=-\infty, n \neq 0}^{\infty}\left(\sum_{k=1}^{m-2} \frac{2}{(\pi i n)^{k}} \frac{G_{m-k}}{(m-k) !}\right) e^{2 \pi i n x}= \begin{cases}\sum_{k=1}^{m-1} \frac{1}{k !(m-k) !} G_{k}(<x>) G_{m-k}(<x>), & \text { for } x \notin \mathbb{Z}, \\ \sum_{k=1}^{m-1} \frac{1}{k !(m-k) !} G_{k} G_{m-k}-\frac{2}{(m-1) !} G_{m-1}, & \text { for } x \in \mathbb{Z} ;\end{cases}
$$

(b)

$$
\begin{aligned}
& -\frac{1}{m !} \sum_{k=1}^{m-2} 2^{k+1}\left(\begin{array}{c}
m \\
k
\end{array}\right) G_{m-k} B_{k}(<x>)=\sum_{k=1}^{m-1} \frac{1}{k !(m-k) !} G_{k}(<x>) G_{m-k}(<x>), \text { for } x \notin \mathbb{Z}, \\
& -\frac{1}{m !} \sum_{k=2}^{m-2} 2^{k+1}\left(\begin{array}{c}
m \\
k
\end{array}\right) G_{m-k} B_{k}(<x>)=\sum_{k=1}^{m-1} \frac{1}{k !(m-k) !} G_{k} G_{m-k}-\frac{2}{(m-1) !} G_{m-1}, \text { for } x \in \mathbb{Z} .
\end{aligned}
$$




\section{The function $\gamma_{m}(<x>)$}

Let $\gamma_{m}(x)=\sum_{k=1}^{m-1} \frac{1}{k(m-k)} G_{k}(x) G_{m-k}(x), \quad(m \geqslant 3)$. Then we will consider the function

$$
\gamma_{m}(<x>)=\sum_{k=1}^{m-1} \frac{1}{k(m-k)} G_{k}(<x>) G_{m-k}(<x>),
$$

defined on $(-\infty, \infty)$, which is periodic with period 1. The Fourier series of $\left.\gamma_{m}(<x\rangle\right)$ is

$$
\sum_{n=-\infty}^{\infty} C_{n}^{(m)} e^{2 \pi i n x}
$$

where

$$
C_{n}^{(m)}=\int_{0}^{1} \gamma_{m}(<x>) e^{-2 \pi i n x} d x=\int_{0}^{1} \gamma_{m}(x) e^{-2 \pi i n x} d x .
$$

Before proceeding further, we note the following

$$
\begin{aligned}
\gamma_{m}^{\prime}(x) & =\sum_{k=1}^{m-1} \frac{1}{k(m-k)}\left(k G_{k-1}(x) G_{m-k}(x)+(m-k) G_{k}(x) G_{m-k-1}(x)\right) \\
& =\sum_{k=2}^{m-1} \frac{1}{m-k} G_{k-1}(x) G_{m-k}(x)+\sum_{k=1}^{m-2} \frac{1}{k} G_{k}(x) G_{m-k-1}(x) \\
& =\sum_{k=1}^{m-2}\left(\frac{1}{m-k-1}+\frac{1}{k}\right) G_{k}(x) G_{m-1-k}(x) \\
& =(m-1) \sum_{k=1}^{m-2} \frac{1}{k(m-1-k)} G_{k}(x) G_{m-1-k}(x) \\
& =(m-1) \gamma_{m-1}(x) .
\end{aligned}
$$

From this, we note that $\left(\frac{\gamma_{m+1}(x)}{m}\right)^{\prime}=\gamma_{m}(x)$,

$$
\begin{aligned}
\int_{0}^{1} \gamma_{m}(x) d x & =\frac{1}{m}\left(\gamma_{m+1}(1)-\gamma_{m+1}(0)\right) \\
\gamma_{m}(1)-\gamma_{m}(0) & =\sum_{k=1}^{m-1} \frac{1}{k(m-k)}\left(G_{k}(1) G_{m-k}(1)-G_{k}(0) G_{m-k}(0)\right) \\
& =\sum_{k=1}^{m-1} \frac{1}{k(m-k)}\left(\left(-G_{k}(0)+2 \delta_{k, 1}\right)\left(-G_{m-k}(0)+2 \delta_{m-k, 1}\right)-G_{k}(0) G_{m-k}(0)\right) \\
& =\sum_{k=1}^{m-1} \frac{1}{k(m-k)}\left(-2 G_{k}(0) \delta_{m-k, 1}-2 \delta_{k, 1} G_{m-k}(0)+4 \delta_{k, 1} \delta_{m-k, 1}\right) \\
& =\frac{1}{m-1}\left(-2 G_{m-1}(0)-2 G_{m-1}(0)+4 \delta_{m, 2}\right) \\
& =-\frac{4}{m-1} G_{m-1} .
\end{aligned}
$$

Thus,

$$
\gamma_{\mathrm{m}}(1)=\gamma_{\mathrm{m}}(0) \Leftrightarrow \mathrm{G}_{\mathrm{m}-1}(0)=0(\mathrm{~m} \geqslant 3) \Leftrightarrow \mathrm{m} \text { is even }(\mathrm{m} \geqslant 3) .
$$


Also,

$$
\int_{0}^{1} \gamma_{m}(x) d x=-\frac{4}{m^{2}} G_{m}
$$

We are now ready to determine the Fourier coefficients $C_{n}^{(m)}$.

Case $1: n \neq 0$.

$$
\begin{aligned}
& C_{n}^{(m)}=\int_{0}^{1} \gamma_{m}(x) e^{-2 \pi i n x} d x \\
& =-\frac{1}{2 \pi i n}\left[\gamma_{m}(x) e^{-2 \pi i n x}\right]_{0}^{1}+\frac{1}{2 \pi i n} \int_{0}^{1} \gamma_{m}^{\prime}(x) e^{-2 \pi i n x} d x \\
& =-\frac{1}{2 \pi i n}\left(\gamma_{m}(1)-\gamma_{m}(0)\right)+\frac{m-1}{2 \pi i n} \int_{0}^{1} \gamma_{m-1}(x) e^{-2 \pi i n x} d x \\
& =\frac{m-1}{2 \pi i n} C_{n}^{(m-1)}+\frac{2}{\pi i n} \frac{G_{m-1}}{m-1} \\
& =\frac{m-1}{2 \pi i n}\left(\frac{m-2}{2 \pi i n} C_{n}^{(m-2)}+\frac{2}{\pi i n} \frac{G_{m-2}}{m-2}\right)+\frac{2}{\pi i n} \frac{G_{m-1}}{m-1} \\
& =\frac{(m-1)(m-2)}{(2 \pi i n)^{2}} C_{n}^{(m-2)}+\frac{m-1}{2 \pi i n} \frac{2}{\pi i n} \frac{G_{m-2}}{m-2}+\frac{2}{\pi i n} \frac{G_{m-1}}{m-1} \\
& =\frac{(m-1)(m-2)}{(2 \pi i n)^{2}}\left(\frac{m-3}{2 \pi i n} C_{n}^{(m-3)}+\frac{2}{\pi i n} \frac{G_{m-3}}{m-3}\right)+\frac{m-1}{2 \pi i n} \frac{2}{\pi i n} \frac{G_{m-2}}{m-2}+\frac{2}{\pi i n} \frac{G_{m-1}}{m-1} \\
& =\frac{(m-1)(m-2)(m-3)}{(2 \pi i n)^{3}} C_{n}^{(m-3)}+\frac{(m-1)(m-2)}{(2 \pi i n)^{2}} \frac{2}{\pi i n} \frac{G_{m-3}}{m-3}+\frac{m-1}{2 \pi i n} \frac{2}{\pi i n} \frac{G_{m-2}}{m-2}+\frac{2}{\pi i n} \frac{G_{m-1}}{m-1} \\
& \vdots \\
& =\frac{(m-1)_{m-3}}{(2 \pi i n)^{m-3}} C_{n}^{(3)}+\sum_{k=1}^{m-3} \frac{(m-1)_{k-1}}{(2 \pi i n)^{k-1}} \frac{2}{\pi i n} \frac{G_{m-k}}{m-k} \\
& =-\frac{2}{m} \frac{(m)_{m-2}}{(2 \pi i n)^{m-2}}+\frac{4}{m} \sum_{k=1}^{m-3} \frac{(m)_{k}}{(2 \pi i n)^{k}} \frac{G_{m-k}}{m-k} \\
& =\frac{4}{m} \sum_{k=1}^{m-2} \frac{(m)_{k}}{(2 \pi i n)^{k}} \frac{G_{m-k}}{m-k}
\end{aligned}
$$

where $C_{n}^{(3)}=\int_{0}^{1} \gamma_{3}(x) e^{-2 \pi i n x} d x=\int_{0}^{1}(2 x-1) e^{-2 \pi i n x} d x=-\frac{1}{\pi i n}$.

Case 2: $n=0$.

$$
C_{0}^{(m)}=\int_{0}^{1} \gamma_{m}(x) d x=-\frac{4}{m^{2}} G_{m}
$$

$\gamma_{m}(<x>), \quad(m \geqslant 3)$ is piecewise $C^{\infty}$. In addition, $\gamma_{m}(<x>)$ is continuous for all even positive integers $m \geqslant 4$ and discontinuous with jump discontinuities at integers for all odd positive integers $m \geqslant 3$.

Assume first that $m$ is an even positive integer with $m \geqslant 4$. Then $\gamma_{m}(1)=\gamma_{m}(0) . \gamma_{m}(<x>)$ is piecewise $C^{\infty}$ and continuous. Hence the Fourier series of $\gamma_{m}(<x>)$ converges uniformly to $\gamma_{m}(<x>)$, and

$$
\gamma_{m}(<x>)=-\frac{4}{m^{2}} G_{m}+\sum_{n=-\infty, n \neq 0}^{\infty}\left(\frac{4}{m} \sum_{k=1}^{m-2} \frac{(m)_{k}}{(2 \pi i n)^{k}} \frac{G_{m-k}}{(m-k)}\right) e^{2 \pi i n x}
$$




$$
\begin{aligned}
& =-\frac{4}{m^{2}} G_{m}-\frac{4}{m} \sum_{k=1}^{m-2}\left(\begin{array}{c}
m \\
k
\end{array}\right) \frac{G_{m-k}}{m-k} \times\left(-k ! \sum_{n=-\infty, n \neq 0}^{\infty} \frac{e^{2 \pi i n x}}{(2 \pi i n)^{k}}\right) \\
& =-\frac{4}{m^{2}} G_{m}-\frac{4}{m} \sum_{k=2}^{m-2}\left(\begin{array}{c}
m \\
k
\end{array}\right) \frac{G_{m-k}}{m-k} B_{k}(<x>)-\frac{4}{m}\left(\begin{array}{c}
m \\
1
\end{array}\right) \frac{G_{m-1}}{m-1} \times \begin{cases}B_{1}(<x>), & \text { for } x \notin \mathbb{Z}, \\
0, & \text { for } x \in \mathbb{Z}\end{cases} \\
& =-\frac{4}{m} \sum_{k=0, k \neq 1}^{m-2}\left(\begin{array}{c}
m \\
k
\end{array}\right) \frac{G_{m-k}}{m-k} B_{k}(<x>) .
\end{aligned}
$$

Hence we have the following theorem.

Theorem 4.1. Let $\mathrm{m}$ be an even integer with $\mathrm{m} \geqslant 4$. Then we have the following:

(a) $\sum_{k=1}^{m-1} \frac{1}{k(m-k)} G_{k}(<x>) G_{m-k}(<x>)$ has the Fourier series expansion

$$
\sum_{k=1}^{m-1} \frac{1}{k(m-k)} G_{k}(<x>) G_{m-k}(<x>)=-\frac{4}{m^{2}} G_{m}+\sum_{n=-\infty, n \neq 0}^{\infty}\left(\frac{4}{m} \sum_{k=1}^{m-2} \frac{(m)_{k}}{(2 \pi i n)^{k}} \frac{G_{m-k}}{m-k}\right) e^{2 \pi i n x}
$$

(b)

for all $x \in(-\infty, \infty)$, where the convergence is uniform;

$$
\sum_{k=1}^{m-1} \frac{1}{k(m-k)} G_{k}(<x>) G_{m-k}(<x>)=-\frac{4}{m} \sum_{k=0, k \neq 1}^{m-2}\left(\begin{array}{c}
m \\
k
\end{array}\right) \frac{G_{m-k}}{m-k} B_{k}(<x>)
$$

for all $\mathrm{x} \in(-\infty, \infty)$, where $\left.\mathrm{B}_{\mathrm{k}}(<\mathrm{x}\rangle\right)$ is the Bernoulli function.

Assume next that $m$ is an odd integer with $m \geqslant 3$. Then, $\gamma_{m}(1) \neq \gamma_{m}(0)$, and hence $\gamma_{m}(<x>)$ is piecewise $\mathrm{C}^{\infty}$ and discontinuous with jump discontinuities at integers. Thus the Fourier series of $\left.\gamma_{\mathrm{m}}(<x\rangle\right)$ convergence pointwise to $\gamma_{\mathrm{m}}(<x>)$ for $x \notin \mathbb{Z}$, and converges to

$$
\frac{1}{2}\left(\gamma_{m}(0)+\gamma_{m}(1)\right)=\gamma_{m}(0)-\frac{2}{(m-1)} G_{m-1}
$$

for $x \in \mathbb{Z}$. Thus we get the following theorem.

Theorem 4.2. Let $\mathrm{m}$ be an odd positive integer with $\mathrm{m} \geqslant 3$. Thus we have the following:

(a)

$$
\sum_{n=-\infty, n \neq 0}^{\infty}\left(\frac{4}{m} \sum_{k=1}^{m-2} \frac{(m)_{k}}{(2 \pi i n)^{k}} \frac{G_{m-k}}{(m-k)}\right) e^{2 \pi i n x}= \begin{cases}\sum_{k=1}^{m-1} \frac{1}{k(m-k)} G_{k}(<x>) G_{m-k}(<x>), & \text { for } x \notin \mathbb{Z}, \\ \sum_{k=1}^{m-1} \frac{1}{k(m-k)} G_{k} G_{m-k}-\frac{2}{(m-1)} G_{m-1}, & \text { for } x \in \mathbb{Z} ;\end{cases}
$$

(b)

$$
\begin{aligned}
& -\frac{4}{m} \sum_{k=1}^{m-2}\left(\begin{array}{c}
m \\
k
\end{array}\right) \frac{G_{m-k}}{m-k} B_{k}(<x>)=\sum_{k=1}^{m-1} \frac{1}{k(m-k)} G_{k}(<x>) G_{m-k}(<x>), \text { for } x \notin \mathbb{Z}, \\
& -\frac{4}{m} \sum_{k=2}^{m-2}\left(\begin{array}{c}
m \\
k
\end{array}\right) \frac{G_{m-k}}{m-k} B_{k}(<x>)=\sum_{k=1}^{m-1} \frac{1}{k(m-k)} G_{k} G_{m-k}-\frac{2}{(m-1)} G_{m-1} \text {, for } x \in \mathbb{Z} .
\end{aligned}
$$

\section{References}

[1] A. Bayad, T. Kim, Identities for the Bernoulli, the Euler and the Genocchi numbers and polynomials, Adv. Stud. Contemp. Math. (Kyungshang), 20 (2010), 247-253. 1

[2] A. Bayad, T. Kim, Identities involving values of Bernstein, q-Bernoulli, and q-Euler polynomials, Russ. J. Math. Phys., 18 (2011), 133-143. 1

[3] A. Bayad, T. Kim, Higher recurrences for Apostol-Bernoulli-Euler numbers, Russ. J. Math. Phys., 19 (2012), 1-10. 1

[4] G. V. Dunne, C. Schubert, Bernoulli number identities from quantum field theory and topological string theory, Commun. Number Theory Phys., 7 (2013), 225-249.1 
[5] C. Faber, R. Pandharipande, Hodge integrals and Gromov-Witten theory, Invent. Math., 139 (2000), 173-199. 1

[6] T. Kim, A note on the q-Genocchi numbers and polynomials, J. Inequal. Appl., 2007 (2007), 8 pages,. 1

[7] T. Kim, On the q-extension of Euler and Genocchi numbers, J. Math. Anal. Appl., 326 (2007), 1458-1465. 1

[8] T. Kim, Euler numbers and polynomials associated with zeta functions, Abstr. Appl. Anal., 2008 (2008), 11 pages. 1

[9] T. Kim, Note on the Euler numbers and polynomials, Adv. Stud. Contemp. Math. (Kyungshang), 17 (2008), 131-136. 1

[10] T. Kim, On the multiple q-Genocchi and Euler numbers, Russ. J. Math. Phys., 15 (2008), 481-486.

[11] T. Kim, Some identities for the Bernoulli, the Euler and the Genocchi numbers and polynomials, Adv. Stud. Contemp. Math. (Kyungshang), 20 (2010), 23-28. 1

[12] D. S. Kim, T. Kim, Bernoulli basis and the product of several Bernoulli polynomials, Int. J. Math. Math. Sci., 2012 (2012), 12 pages. 1

[13] D. S. Kim, T. Kim, Euler basis, identities, and their applications, Int. J. Math. Math. Sci., 2012 (2012), 15 pages.

[14] D. S. Kim, T. Kim, A note on higher-order Bernoulli polynomials, J. Inequal. Appl., 2013 (2013), 9 pages.

[15] D. S. Kim, T. Kim, Some identities of higher order Euler polynomials arising from Euler basis, Integral Transforms Spec. Funct., 24 (2013), 734-738.

[16] D. S. Kim, T. Kim, Identities arising from higher-order Daehee polynomial bases, Open Math., 13 (2015), 196-208. 1

[17] T. Kim, D. S. Kim, S.-H. Rim, D. V. Dolgy, Fourier series of higher-order Bernoulli functions and their applications, J. Inequal. Appl., 2017 (2017), 7 pages. 1

[18] Q.-M. Luo, Extensions of the Genocchi polynomials and their Fourier expansions and integral representations, Osaka J. Math., 48 (2011), 291-309. 1

[19] J. E. Marsden, Elementary classical analysis, With the assistance of Michael Buchner, Amy Erickson, Adam Hausknecht, Dennis Heifetz, Janet Macrae and William Wilson, and with contributions by Paul Chernoff, István Fáry and Robert Gulliver. W. H. Freeman and Co., San Francisco, (1974). 1

[20] K. Shiratani, S. Yokoyama, An application of p-adic convolutions, Mem. Fac. Sci. Kyushu Univ. Ser. A, 36 (1982), 73-83. 1

[21] H. M. Srivastava, Some generalizations and basic (or q-) extensions of the Bernoulli, Euler and Genocchi polynomials, Appl. Math. Inf. Sci., 5 (2011), 390-414. 1

[22] D. Zill, M. R. Cullen, Advanced engineering mathematics, Second edition, Jones and Bartlett, Massachusetts, (2006). 